\title{
FUNCTIONS OF UNIFORMLY BOUNDED CHARACTERISTIC ON RIEMANN SURFACES
}

\author{
BY
}

SHINJI YAMASHITA

\begin{abstract}
A characteristic function $T(D, w, f)$ of Shimizu and Ahlfors type for a function $f$ meromorphic in a Riemann surface $R$ is defined, where $D$ is a regular subdomain of $R$ containing a reference point $w \in R$. Next we suppose that $R$ has the Green functions. Letting $T(w, f)=\lim _{D \uparrow R} T(D, w, f)$, we define $f$ to be of uniformly bounded characteristic in $R, f \in \operatorname{UBC}(R)$ in notation, if $\sup _{w \in R} T(w, f)<\infty$. We shall propose, among other results, some criteria for $f$ to be in $\operatorname{UBC}(R)$ in various terms, namely, Green's potentials, harmonic majorants, and counting functions. They reveal that $\operatorname{UBC}(\Delta)$ for the unit disk $\Delta$ coincides precisely with that introduced in our former work. Many known facts on $\operatorname{UBC}(\Delta)$ are extended to $\operatorname{UBC}(R)$ by various methods. New proofs even for $R=\Delta$ are found. Some new facts, even for $\Delta$, are added.
\end{abstract}

0. Introduction. We shall extend the notion of $\mathrm{UBC}$ and $\mathrm{UBC}_{0}$ from the unit disk $\Delta=\{|z|<1\}$ (see $\left[\mathbf{Y}_{\mathbf{1}}\right.$ and $\left.\mathbf{Y}_{\mathbf{2}}\right]$ ) to hyperbolic Riemann surfaces, prove some results analogous to those in $\Delta$, and add some facts, new, even for $\Delta$. A hyperbolic Riemann surface $S$ is one possessing Green functions; thus, its universal covering surface $S^{\infty}$ must be conformally equivalent to $\Delta$, so that $S^{\infty}$ and $\Delta$ are identified.

Our study begins with how to define the Shimizu-Ahlfors characteristic function $T(D, w, f)$ on "good" subdomains $D$ containing a point $w$ of a Riemann surface $R$, hyperbolic or not, on which $f$ is meromorphic. Each point of $R$ is identified with its local-parametric image in the complex plane $\mathbf{C}=\{|z|<\infty\}$. By $D$ we always mean a relatively compact subdomain of $R$, whose boundary $\partial D$ consists of a finite number of mutually disjoint, analytic, simple and closed curves on $R$. If we refer to a pair $D$ and $w \in R$ we always assume that $w \in D$. The radius $r=r(D, w)>0$ of $D$ is defined by

$$
\log r=\lim \left(g_{D}(z, w)+\log |z-w|\right)
$$

as $z \rightarrow w$ within the parametric disk of center $w$, where $g_{D}(z, w)$ is the Green function of $D$ with pole at $w$. We now set

$$
T(D, w, f)=\pi^{-1} \int_{0}^{r} t^{-1}\left[\iint_{D_{t}} f^{\#}(z)^{2} d x d y\right] d t
$$

where $D_{t}=\left\{z \in D ; g_{D}(z, w) \geq \log (r / t)\right\}, 0<t<r$, and

$$
f^{\#}(z)= \begin{cases}\left|f^{\prime}(z)\right| /\left(1+|f(z)|^{2}\right), & \text { if } f(z) \neq \infty \\ (1 / f)^{\#}(z), & \text { if } f(z)=\infty\end{cases}
$$

Received by the editors June 6, 1983 and, in revised form, May 10, 1984.

1980 Mathematics Subject Classification. Primary 30D30, 30D50, 30F99; Secondary 30F 15. 
is not a function on $R$, yet the second-order differential $f^{\#}(z)^{2} d x d y, z=x+i y \in$ $R$, is well defined on $R$. The Green-potential expression

$$
T\left(D, u^{\prime}, f\right)=\pi^{-1} \iint_{D} f^{\#}(z)^{2} g_{D}(z, w) d x d y, \quad w \in R,
$$

will be proved later. The nomenclature of $T$ is justified because for $R=\{|z|<$ $\rho\}, D=\{|z|<r\}$ with $0<r<\rho \leq \infty$, and $w=0$, we have the usual one because $g_{D}(z, 0)=\log |r / z|$.

Henceforth we always assume that $R$ is hyperbolic and we set

$$
T(w, f)=T(R, w, f)=\lim _{D \uparrow R} T(D, w, f) \leq \infty .
$$

This means that given $\varepsilon>0$ we can find a compact set $K, w \in K \subset R$, such that $|T-T(D)|<\varepsilon$ for all $D \supset K$, with the obvious change in case $T=\infty$. Lebesgue's convergence theorem applied to $(0.2)$ yields

$$
T(w, f)=\pi^{-1} \iint_{R} f^{\#}(z)^{2} g(z, w) d x d y
$$

where $g=g_{R}$ is the Green function on $R ;(0.2)$ can be regarded as the case $R=D$.

A meromorphic $f$ on $R$ is said to be of uniformly bounded characteristic, $f \in$ $\mathrm{UBC} \equiv \operatorname{UBC}(R)$ in notation, if the function $T(w, f)$ is bounded on $R$, while, $f \in \mathrm{UBC}_{0} \equiv \mathrm{UBC}_{0}(R)$ if $\lim _{w \rightarrow \partial R} T(w, f)=0$, that is, for $\varepsilon>0$ there exists a compact $K \subset R$ such that $T(w, f)<\varepsilon$ in $R \backslash K$.

In $\S 1$ we extend our study from the family $M \equiv M(R)$ of meromorphic functions on $R$ to $M_{e} \equiv M_{e}(R)$ consisting of multiple-valued meromorphic functions with single-valued moduli on $R$. We can easily extend the definition of UBC $\left(\mathrm{UBC}_{0}\right.$, respectively) for $f \in M$ to $\mathrm{UBC}_{e} \equiv \mathrm{UBC}_{e}(R)\left(\mathrm{UBC}_{e 0} \equiv \mathrm{UBC}_{e 0}(R)\right.$, resp.) for $f \in M_{e}$.

In $\S 2,(0.3)$ for $f \in M_{e}$ is proved. Thus, criteria are obtained in terms of the Green potentials (Corollary 2.2). The families $\mathrm{BMOA}_{e} \equiv \mathrm{BMOA}_{e}(R)$ and $\mathrm{VMOA}_{e} \equiv \mathrm{VMOA}_{e}(R)$ are defined for pole-free members of $M_{e}$; these are extensions of $\mathrm{BMOA}$ and VMOA in the disk. For the definition of $\mathrm{BMOA}(R)$ see $[\mathbf{M}]$; note that $\mathrm{BMOA}(R)=M(R) \cap \mathrm{BMOA}_{e}(R)$. The formulae $\mathrm{BMOA}_{e} \subset \mathrm{UBC}_{e}$ and $\mathrm{VMOA}_{e} \subset \mathrm{UBC}_{e 0}$ are now obvious. An expression of $T$ in terms of the limit $(D \uparrow R)$ of the mean of $\frac{1}{2} \log \left(1+|f|^{2}\right)$ on $\partial D$ and the limit of

$$
N(D, w, f)=\sum_{f(b)=\infty, b \in D} g_{D}(w, b)
$$

will be of use to compare $T$ with L. Sario's characteristic function $T_{S}$ (see [SN]). Sario's class $M_{e} B(R)$ coincides with that of $f \in M_{e}$ for which $T(w, f)$ is finite for a $w=w(f) \in R$.

$\S 3$ is devoted to the study of the least harmonic majorant $\varphi^{\wedge}$ of

$$
\varphi=\frac{1}{2} \log \left(\left|f_{1}\right|^{2}+\left|f_{2}\right|^{2}\right) \quad \text { for } f \in M_{e},
$$

where $|f|=\left|f_{1}\right| /\left|f_{2}\right|, f_{1}, f_{2} \in M_{e}$, is an "admissible" decomposition. A new expression $T(w, f)=\varphi^{\wedge}(w)-\varphi(w), w \in R$, is of use to obtain criteria for $f \in M_{e}$ to be of $\mathrm{UBC}_{e}$ and of $\mathrm{UBC}_{e 0}$ in terms of $\varphi^{\wedge}-\varphi$. Some remarks refer to strong parallels between $\mathrm{BMOA}_{e}\left(\mathrm{VMOA}_{e}\right.$, resp.) and $\mathrm{UBC}_{e}\left(\mathrm{UBC}_{e 0}\right.$, resp.). 
In $\S 4$, criteria for $f \in M$ to be of UBC in terms of the supremum of the function $N(z)=\lim _{D \uparrow R} N(D, w, 1 /(f-z)), z \in \mathbf{C}^{*}=\mathbf{C} \cup\{\infty\}$, on the spherical circle of center $f(w)$ are obtained.

The projection $\pi: \Delta \rightarrow R$ is considered in $\S 5$, and the identity $T(R, \pi(\delta), f)=$ $T(\Delta, \delta, f \circ \pi), \delta \in \Delta$, for $f \in M(R)$ is proved. As applications we obtain: (1) If $f \in \operatorname{UBC}(S)$ and $h: R \rightarrow S$ is an analytic map, then $f \circ h \in \operatorname{UBC}(R)$. (2) If $h: R \rightarrow S$ is of type $B l$ in the sense of M. Heins $\left[\mathbf{H}_{\mathbf{1}}\right]$, and if $f \circ h \in \operatorname{UBC}(R)$, then $f \in \mathrm{UBC}(S)$. Finally, a contribution is made to the classification of Riemann surfaces: $O_{\mathrm{UBC}} \varsubsetneqq O_{\mathrm{BMOA}}$.

1. Families $\mathrm{UBC}_{e}$ and $\mathrm{UBC}_{e 0}$. The functions on $R$, which we shall actually study, are, for the most part, the "generalized" meromorphic functions on $R$. Let $M_{e} \equiv M_{e}(R)$ be the family of multiple-valued functions $f=\exp \left(u+i u^{*}\right)$ on $R$, where $u$ is a single-valued function harmonic on $R$ except for countably many logarithmic singularities $a_{n}$ clustering nowhere in $R$, such that $u(z)-k_{n} \log \left|z-a_{n}\right|$ is harmonic in the parameter disk of center $a_{n}$ with the integral coefficient $k_{n}$. The multiple-valuedness of $f$ arises from that of the conjugate function $u^{*}$ of $u$ on $R$. It is natural to regard the constant zero as a member of $M_{e}$.

The modulus $|f|$ of $f \in M_{e}$ is single-valued throughout $R$, and each branch of nonconstant $f$ in the parametric disk of each point $w \in R$ is single-valued there, and has the Laurent expansion

$$
c_{\lambda}(z-w)^{\lambda}+c_{\lambda+1}(z-w)^{\lambda+1}+\cdots,
$$

where $\lambda$ is an integer with $c_{\lambda} \neq 0$; the branches differ by multiplicative constants of moduli one. Therefore $\left|c_{\lambda}\right|$ is definite and

$$
|f(w)| \leq\left|c_{\lambda}\right| \text { if }|f(w)| \neq \infty .
$$

We call $w \in R$ a zero of $f$ of order $\lambda$ if $|f(w)|=0$. Similarly, $w \in R$ is a pole (or, $\infty$-point) of $f$ of order $-\lambda$ if $|f(w)|=\infty$.

The family $M \equiv M(R)$ of single-valued members of $M_{e}$ consists of all the meromorphic functions on $R$. For $a \in \mathbf{C}$ we call $w \in R$ an $a$-point of order $\lambda$ of $f \in M$ if $w$ is a zero of order $\lambda$ of $f-a$.

It is now easy to extend $T(D, w, f)$ and $T(w, f) \equiv T(R, w, f)$ to $f \in M_{e}$. Actually, $\left|f^{\prime}(z)\right|$ for $|f(z)| \neq \infty$, as well as $f^{\#}(z)$ defined by $(0.1)$, is definite, so that the differentials $\left|f^{\prime}(z)\right|^{2} d x d y$ for pole-free $f$ and $f^{\#}(z)^{2} d x d y$ for arbitrary $f$ are defined on $R$. The definitions of $\mathrm{UBC}_{e} \equiv \mathrm{UBC}_{e}(R)$ and $\mathrm{UBC}_{e 0} \equiv \mathrm{UBC}_{e 0}(R)$ are thus clear; just extend those of $\mathrm{UBC} \equiv \mathrm{UBC}(R)$ and $\mathrm{UBC}_{0} \equiv \mathrm{UBC}_{0}(R)$ in the introduction to $f \in M_{e}$.

2. The Shimizu-Ahlfors characteristic function. We begin with the Green-potential expression (0.3) for $f \in M_{e}$.

THEOREM 2.1. The identity

$$
T(w, f)=\pi^{-1} \iint_{R} f^{\#}(z)^{2} g(z, w) d x d y \quad(\leq \infty)
$$

holds for each $f \in M_{e}(R)$ and each $w \in R$.

PROOF. It suffices to establish

$$
T(D, w, f)=\pi^{-1} \iint_{D} f^{\#}(z)^{2} g_{D}(z, w) d x d y
$$


for each pair $D, w$. Set

$$
c_{t}(z)= \begin{cases}1, & \text { if } z \in D_{t} \\ 0, & \text { if } z \in D \backslash D_{t}\end{cases}
$$

Then the identity

$$
\int_{0}^{r} c_{t}(z) t^{-1} d t=g_{D}(z, w), \quad z \in D
$$

together with

$$
T(D, w, f)=\pi^{-1} \iint_{D}\left[\int_{0}^{r} c_{t}(z) t^{-1} d t\right] f^{\#}(z)^{2} d x d y
$$

proves (2.2).

REMARK. Suppose that $w$ is in the parametric disk $U_{w^{\prime}}$ of center $w^{\prime} \in R$, and define $r^{\prime}>0$ by

$$
\log r^{\prime}=\lim \left(g_{D}(z, w)+\log |z-w|\right),
$$

where, this time $z \rightarrow w$ within $U_{w^{\prime}}$. The same proof as above then shows that

$$
T(D, w, f)=\pi^{-1} \int_{0}^{r^{\prime}} t^{-1}\left[\iint_{D_{t}^{\prime}} f^{\#}(z)^{2} d x d y\right] d t,
$$

where $D_{t}^{\prime}=\left\{z \in D ; g_{D}(z, w) \geq \log \left(r^{\prime} / t\right)\right\}, 0<t<r^{\prime}$.

Two corollaries follow from Theorem 2.1.

COROLlary 2.2. For $f \in M_{e}(R)$ the following are valid.

(I) $f \in \mathrm{UBC}_{e}(R)$ if and only if

$$
\sup _{w \in D} \iint_{R} f^{\#}(z)^{2} g(z, w) d x d y<\infty .
$$

(II) $f \in \mathrm{UBC}_{e 0}(R)$ if and only if

$$
\lim _{w \rightarrow \partial R} \iint_{R} f^{\#}(z)^{2} g(z, w) d x d y=0 .
$$

This corollary extends [ $\mathbf{Y}_{\mathbf{1}}$, Theorem 2.2, p. 352].

A pole-free $f \in M_{e}(R)$ is said to be of bounded (vanishing, resp.) mean oscillation on $R, f \in \mathrm{BMOA}_{e}(R)\left(f \in \mathrm{VMOA}_{e}(R)\right.$, resp.) in notation, if

$$
\begin{aligned}
\sup _{w \in R} \iint_{R}\left|f^{\prime}(z)\right|^{2} g(z, w) d x d y & <\infty \\
& \left(\lim _{w \rightarrow \partial R} \iint_{R}\left|f^{\prime}(z)\right|^{2} g(z, w) d x d y=0 \text {, resp. }\right) .
\end{aligned}
$$

The family $\operatorname{BMOA}(R)=\mathrm{BMOA}_{e}(R) \cap M(R)$ is introduced by $\mathrm{T}$. A. Metzger $[\mathbf{M}]$. An immediate consequence of Corollary 2.2 is the following which extends $\left[\mathbf{Y}_{\mathbf{1}}\right.$, Theorem 7.1, p. 364].

Corollary 2.3. $\operatorname{BMOA}_{e}(R) \subset \operatorname{UBC}_{e}(R)$ and $\operatorname{VMOA}_{e}(R) \subset \mathrm{UBC}_{e 0}(R)$.

Following Sario we shall define the proximity function $m_{S}(D, w, f)$, the counting function $N_{S}(D, w, f)$, and the characteristic function $T_{S}(D, w, f)$ for $f \in M_{e}$ and $w \in D$. They are extensions of M. Parreau's [P, p. $183 \mathrm{ff}$. $]$ for $f \in M$. The reader is expected to be familiar with $\left[\mathbf{S N}\right.$, Chapter III] or with the papers $\left[\mathbf{S}_{\mathbf{1}}\right.$ and $\left.\mathbf{S}_{\mathbf{2}}\right]$. 
Let $\log ^{+} x=\max (\log x, 0)$ for $0 \leq x \leq \infty$, and set

$$
m_{S}(D, w, f)=-(2 \pi)^{-1} \int_{\partial D} \log ^{+}|f(z)| d g_{D}^{*}(z, w),
$$

where $\partial D$ is oriented positively with respect to $D$, and the star means the conjugate. The comparison

$$
m_{S}(D, w, f) \leq m(D, w, f) \leq \frac{1}{2} \log 2+m_{S}(D, w, f)
$$

of $m_{S}(D, w, f)$ with

$$
m(D, w, f)=-(4 \pi)^{-1} \int_{\partial D} \log \left(1+|f(z)|^{2}\right) d g_{D}^{*}(z, w)
$$

will be of use; $(2.5)$ is a consequence of

$$
\log ^{+} x \leq \frac{1}{2} \log \left(1+x^{2}\right) \leq \frac{1}{2} \log 2+\log ^{+} x \text { for } 0 \leq x \leq \infty .
$$

For $0<t<r$, let $n(t, f)$ be the number of the poles of $f$, counted with the orders, in $D_{t}$. We define

$$
N_{S}(D, w, f)=\int_{0}^{r} t^{-1}[n(t, f)-n(0, f)] d t+n(0, f) \log r
$$

where $n(0, f)=\lim _{t \rightarrow+0} n(t, f)$. Set $N(D, w, f)=\sum g_{D}(w, b)$, where the sum is extended over all the poles $b$ of $f$ in $D$, each counted with its order. If $f$ is pole-free in $D$, then $N_{S}=N=0$. A routine procedure [SN, p. 76] yields that $N_{S}(D, w, f)=N(D, w, f)$ in case $|f(w)| \neq \infty$. Therefore

$$
N(D, w, f)=\int_{0}^{r} t^{-1} n(t, f) d t
$$

for all $w \in D$ because $N(D, w, f)=\infty$ if $|f(w)|=\infty$. The characteristic function for $f$ is now defined by

$$
T_{S}(D, w, f)=m_{S}(D, w, f)+N_{S}(D, w, f) .
$$

For $X=m, N, m_{S}, N_{S}$, and $T_{S}$, we set $X(w, f)=\lim _{D \uparrow R} X(D, w, f)$.

We shall compare $T_{S}(w, f)$ with $T(w, f)$ in Corollary 2.5 below.

THEOREM 2.4. If $|f(w)| \neq \infty$ for $f \in M_{e}(R)$, then

$$
T(w, f)=m(w, f)+N(w, f)-\frac{1}{2} \log \left(1+|f(w)|^{2}\right) .
$$

If $|f(w)|=\infty$, then

$$
T(w, f)=m(w, f)+N_{S}(w, f)-\log \left|c_{\lambda}\right|
$$

where $c_{\lambda}$ is defined in (1.1).

REMARK. If $f \in M_{e}(R)$ is bounded, $|f| \leq K$, then $T(w, f) \leq \frac{1}{2} \log \left(1+K^{2}\right)$ by (2.7), so that $f \in \mathrm{UBC}_{e}(R)$.

COROLlaRY 2.5. If $f \in M_{e}(R)$ is nonconstant, then

$$
\left|T(w, f)-T_{S}(w, f)\right| \leq \frac{1}{2} \log 2+|\log | c_{\lambda}|| .
$$

Read (2.9), in the specified case, as $T(w, f)=\infty$ if and only if $T_{S}(w, f)=\infty$.

Fix $w \in R$ and let $\mathrm{BC}_{e} \equiv \mathrm{BC}_{e}(R)$ be the family of $f \in M_{e}$ such that $T_{S}(w, f)<$ $\infty$. As will be observed later in Remark (a) after Theorem $3.1, \mathrm{BC}_{e}$ does not depend on $w$. Note that $\mathrm{BC}_{e}(R)=M_{e} B(R)$ in $[\mathbf{S N}$, p. 78]; we let $\mathrm{BC}(R)=$ $\mathrm{BC}_{e}(R) \cap M(R)$. An immediate consequence is the following. 
Corollary 2.6. $\mathrm{UBC}_{e}(R) \subset \mathrm{BC}_{e}(R)$.

Hereafter, mainly in the proofs, we shall frequently use the following abbreviations:

$$
X(f)=X(D, w, f) \quad \text { for } X=m, N, T, m_{S}, N_{S}, T_{S} .
$$

PROOF OF THEOREM 2.4. It suffices to prove

$$
\begin{gathered}
T(f)=m(f)+N(f)-\frac{1}{2} \log \left(1+|f(w)|^{2}\right), \quad \text { if }|f(w)| \neq \infty \\
T(f)=m(f)+N_{S}(f)-\log \left|c_{\lambda}\right|, \quad \text { if }|f(w)|=\infty
\end{gathered}
$$

Suppose first that no pole of $f$ lies on $\partial D$, and let $b$ be all the distinct poles of $f$ with orders $k(b)$ in $D$. For sufficiently small $\varepsilon>0$, we let $\gamma_{w}=\{|z-w| \leq \varepsilon\}$ and $\gamma_{b}=\{|z-b| \leq \varepsilon\}$. Apply the Green formula to the function $\psi=(1 / 2) \log \left(1+|f|^{2}\right)$ on the domain $D_{\varepsilon}=D \backslash \gamma_{w} \backslash \bigcup_{b} \gamma_{b}$. Since

$$
\Delta \psi=\left(\partial^{2} / \partial x^{2}+\partial^{2} / \partial y^{2}\right) \psi=2 f^{\# 2} \quad \text { in } D_{\varepsilon},
$$

it follows that

$$
\begin{aligned}
\iint_{D_{\varepsilon}} g_{D}(z, w) & \Delta \psi(z) d x d y \\
= & -\int_{\partial D_{\varepsilon}} g_{D}(z, w) \frac{\partial \psi(z)}{\partial \nu}|d z|+\int_{\partial D_{\varepsilon}} \psi(z) \frac{\partial g_{D}(z, w)}{\partial \nu}|d z|
\end{aligned}
$$

where the normal derivatives $\partial / \partial \nu$ are considered in the direction of the inner normal. As to the first integral in the right-hand side of (2.11), that on $\partial D$ is zero, and those on $\partial \gamma_{w}$ and $\partial \gamma_{b}$ tend to zero and $2 \pi k(b) g_{D}(b, w)$ as $\varepsilon \rightarrow 0$, respectively. Furthermore, as to the second, that on $\partial D$ equals $2 \pi m(f)$, and those on $\partial \gamma_{w}$ and $\partial \gamma_{b}$ tend to $-2 \pi \psi(w)$ and 0 as $\varepsilon \rightarrow 0$, respectively. The resulting identity divided by $2 \pi$, together with $g_{D}(b, w)=g_{D}(w, b)$, yields

$$
\pi^{-1} \iint_{D} g_{D}(z, w) f^{\#}(z)^{2} d x d y=m(f)-\psi(w)+\sum_{b} k(b) g_{D}(w, b) .
$$

In view of $(2.2)$ we immediately observe that $\left(2.7^{\prime}\right)$ is true.

Suppose now that $\partial D$ contains at least one pole of $f$. For $t, 0<t<r$, sufficiently near $r$, we obtain $\left(2.7^{\prime}\right)$ for $D_{t} \backslash \partial D_{t}$ instead of $D$. Observing that $T, m$, and hence $N$, all are continuous in $t$, one obtains $\left(2.7^{\prime}\right)$ for $D$ by letting $t \uparrow r$.

For the proof of $\left(2.8^{\prime}\right)$ we quote Jensen's formula

$$
\log \left|c_{\lambda}\right|=T_{S}(f)-T_{S}(1 / f)
$$

valid for $f$ without any assumption on $|f(w)|$ (see [SN, (7), p. 77].).

Now, for $f$ with $|f(w)|=\infty$, we first note that $\left(2.7^{\prime}\right)$ for $1 / f$ yields

$$
N_{S}(1 / f)=N(1 / f)=T(1 / f)-m(1 / f)=T(f)-m(1 / f)
$$

On the other hand, (2.13) for the present $f$, together with $\log |f|=\log ^{+}|f|-$ $\log ^{+}|1 / f|$, yields.

$$
\log \left|c_{\lambda}\right|=-(2 \pi)^{-1} \int_{\partial D} \log |f(z)| d g_{D}^{*}(z, w)+N_{S}(f)-N_{S}(1 / f),
$$


so that, by $(2.14)$,

$$
\begin{aligned}
& =-(2 \pi)^{-1} \int_{\partial D} \log |f(z)| d g_{D}^{*}(z, w)+N_{S}(f)-T(f)+m(1 / f) \\
& =N_{S}(f)-T(f)+m(f)
\end{aligned}
$$

which completes the proof of $\left(2.8^{\prime}\right)$.

PROOF OF COROLLARY 2.5. We again use (2.10). It suffices to prove

$$
\left|T(f)-T_{S}(f)\right| \leq \frac{1}{2} \log 2+|\log | c_{\lambda}||,
$$

which yields (2.9) on letting $D \uparrow R$. In the case $|f(w)| \neq \infty$, it follows from $N_{S}(f)=N(f)$, the inequalities (2.5) and (2.6) for $x=|f(w)|$, and $\left(2.7^{\prime}\right)$, that

$$
\left|T(f)-T_{S}(f)+\log ^{+}\right| f(w)|| \leq \frac{1}{2} \log 2 .
$$

As is observed in (1.2), $|f(w)| \leq\left|c_{\lambda}\right|$, so that (2.15) now follows from (2.16). In the case $|f(w)|=\infty$, we apply $(2.16)$ to $1 / f$ instead of $f$, to obtain

$$
\left|T(f)-T_{S}(1 / f)\right|=\left|T(1 / f)-T_{S}(1 / f)\right| \leq \frac{1}{2} \log 2 .
$$

It then follows from (2.13) that

$$
\left|T(f)-T_{S}(f)+\log \right| c_{\lambda}|| \leq \frac{1}{2} \log 2,
$$

whence (2.15).

3. An admissible and the canonical decompositions. For $f \in M_{e}(R)$ we call

$$
|f|=\left|f_{1} / f_{2}\right| \text { on } R
$$

an admissible decomposition if $f_{k} \in M_{e}$ is pole-free $(k=1,2)$ and furthermore if both have no common zero, or,

$$
\left|f_{1}\right|^{2}+\left|f_{2}\right|^{2}>0 \text { on } R
$$

This decomposition is not unique.

LEMMA 3.0. Each $f \in M_{e}(R)$ has an admissible decomposition (3.1), where one of $f_{1}$ and $f_{2}$ is a member of $M(R)$.

PROOF. If $f$ is pole-free, then set $f_{1} \equiv f$ and $f_{2} \equiv 1$. If $f$ has one pole at least, then by H. Florack's theorem [F, Satz II, pp. 3-4], there exists a pole-free $f_{2} \in M$ such that $z \in R$ is a zero of order $k$ of $f_{2}$ if and only if $z$ is a pole of order $k$ of $f$. We now obtain (3.1) on setting $f_{1}=f f_{2}$. We note that if $f$ is zero-free, then set $f_{1} \equiv 1$ and $f_{2}=1 / f$. If $f$ has one zero at least, then there exists a pole-free $h_{1} \in M$ such that $z \in R$ is a zero of order $k$ of $h_{1}$ if and only if $z$ is a zero of order $k$ of $f$. We then obtain another admissible decomposition $|f|=\left|h_{1} / h_{2}\right|$ for $h_{2}=h_{1} / f$.

By a harmonic majorant of a subharmonic function $v$ on $R$ we mean a function $u$ harmonic and $v \leq u$ on $R$. If $v$ has a harmonic majorant on $R$, then it has the least harmonic majorant $v^{\wedge}=v_{R}^{\wedge}$ in the sense that $v^{\wedge}$ is a harmonic majorant of $v$ and $v^{\wedge} \leq u$ for each harmonic majorant $u$ of $v$ on $R$.

For an admissible decomposition (3.1) for $f \in M_{e}(R)$ we denote

$$
\varphi=\frac{1}{2} \log \left(\left|f_{1}\right|^{2}+\left|f_{2}\right|^{2}\right) \quad \text { on } R \text {. }
$$


This is a finite-valued subharmonic function on $R$ because (3.2) holds and

$$
\Delta \varphi(z) d x d y=2 f^{\#}(z)^{2} d x d y \quad \text { on } R .
$$

Therefore, for $\varphi_{1}=\frac{1}{2} \log \left(\left|F_{1}\right|^{2}+\left|F_{2}\right|^{2}\right)$ for another admissible decomposition $|f|=$ $\left|F_{1} / F_{2}\right|, \varphi-\varphi_{1}$ is a harmonic function on $R$; thus $\varphi$ is unique modulo harmonic functions on $R$.

THEOREM 3.1. Suppose that $f \in M_{e}(R)$. Then $f \in \mathrm{BC}_{e}(R)$ if and only if $\varphi$ has a harmonic majorant on $R$. If $f \in \mathrm{BC}_{e}(R)$, then

$$
T(w, f)=\varphi_{R}^{\wedge}(w)-\varphi(w) \quad \text { for each } w \in R,
$$

so that the function $\varphi_{R}^{\wedge}-\varphi$ is independent of a choice of decomposition (3.1).

REMARKS. (a) It follows from Corollary 2.5, together with (3.5) that if $f \in$ $\mathrm{BC}_{e}(R)$, then $T_{S}(w, f)<\infty$ for every $w \in R$. Consequently, $\mathrm{BC}_{e}$ does not depend on a choice of $w \in R$.

(b) If $f \in \mathrm{BC}_{e}(R)$, then (3.5) shows that $T(z, f)$ is a $C^{\infty}$ function of real variables $x, y, z=x+i y$, together with

$$
\Delta T(z, f) d x d y=-2 f^{\#}(z)^{2} d x d y
$$

by (3.4). This answers the question raised in [ $\mathbf{Y}_{\mathbf{1}}$, Remark (b), p. 361]. Note that $\left[\mathbf{Y}_{\mathbf{1}}\right.$, Theorem 5.1, p. 360] is a specified case of Theorem 3.1 for pole-free $f \in M(\Delta)$.

As an immediate consequence we obtain

COROLlary 3.2. Suppose that $f \in M_{e}(R)$. Then $f \in \mathrm{UBC}_{e}(R)$ if and only if $\varphi$ has a harmonic majorant on $R$ and $\varphi_{R}^{\wedge}-\varphi$ is bounded there. Further, $f \in$ $\mathrm{UBC}_{e 0}(R)$ if and only if $\varphi$ has a harmonic majorant on $R$ and

$$
\lim _{w \rightarrow \partial R}\left[\varphi_{R}^{\wedge}(w)-\varphi(w)\right]=0 .
$$

The inclusion formula $\mathrm{UBC}_{e 0}(R) \subset \mathrm{UBC}_{e}(R)$ is now obvious. The proof of $\left[\mathbf{Y}_{\mathbf{1}}\right.$, Lemma 2.1, p. 352] is thus facilitated. In fact, this is an immediate consequence of continuity of $T(w, f)$ observed in Remark (b) above.

For clarity we propose

Corollary 3.3. For $f \in M_{e}(R)$ to be in $\mathrm{UBC}_{e}(R)$ it is necessary and sufficient that $\lim \sup _{w \rightarrow \partial R} T(w, f)<\infty$.

More precisely, there exists a compact set $K \subset R$ such that $T(w, f)$ is bounded in $R \backslash K$.

As the third corollary of Theorem 3.1 we claim that a compact set of capacity zero on $R$ is removable for functions of UBC. A set $E \subset R$ is said to be of capacity zero if the intersection of $E$ with the parameter disk at each point of $E$ is of capacity zero [T, p. 55] in C. If $E$ is closed further, then $E$ is totally disconnected, so that $R \backslash E$ is connected. The following is never obvious and needs a proof.

COROLLARY 3.4. Let $E$ be a compact set of capacity zero on $R$ and suppose that $f \in \operatorname{UBC}(R \backslash E)$. Then there exists $F \in \operatorname{UBC}(R)$ such that $F \equiv f$ in $R \backslash E$.

REMARK. If $f \in \mathrm{UBC}(R \backslash E)$ is pole-free, then, is $F$ pole-free? The answer is "no". Just let $f(z)=1 / z, R=\Delta$, and $E=\{0\}$. 
Proof OF THEOREM 3.1. Let $w$ be the point in the definition of $\mathrm{BC}_{e}(R)$. We use the device (2.10). Since

$$
\varphi_{D}^{\wedge}(w)=-(2 \pi)^{-1} \int_{\partial D} \varphi(z) d g_{D}^{*}(z, w)
$$

it follows from (2.2) and (3.4), together with the Green formula, that

$$
2 \pi T(f)=\iint_{D}(\Delta \varphi(z)) g_{D}(z, w) d x d y=-\int_{\partial D} \varphi(z) d g_{D}^{*}(z, w)-2 \pi \varphi(w),
$$

so that

$$
T(f)=\varphi_{D}^{\wedge}(w)-\varphi(w) .
$$

Thus, $\lim _{D \uparrow R} \varphi_{D}^{\wedge}(w)<\infty$ if and only if $T_{S}(w, f)<\infty$, by (2.9). Now, if $\varphi_{R}^{\wedge}$ exists, then (3.5) follows because the same calculation shows that (3.6) is true for each $w \in R$.

ProOF OF COROllary 3.4. We may assume that $f$ is nonconstant. First of all, $\mathrm{BC}(R)$ coincides with Parreau's class $\left(A M_{0}\right)$ in $R[\mathbf{P}$, Definition 1, p. 180]; compare $[\mathbf{P}$, Théorème $19, \mathrm{a}), \mathrm{p} .181]$ with $[\mathbf{S N}$, Theorem $3 \mathrm{~B},(33), \mathrm{p} .83]$. By the case $\alpha=0$ in $\left[\mathbf{P}\right.$, Théorème 20, p. 182] there exists $F \in \mathrm{BC}(R)\left(F=f_{1}\right.$ in Parreau's proof) with $F \equiv f$ in $R \backslash E$. An admissible decomposition $F=f_{1} / f_{2}$ of $F$ in $R$ yields that of $f$ in $R \backslash E$ also. Thus, for $\varphi$ of (3.3) for $F$,

$$
\varphi_{R}^{\wedge}-\varphi=\left(\varphi_{R}^{\wedge}-\varphi_{R \backslash E}^{\wedge}\right)+\left(\varphi_{R \backslash E}^{\wedge}-\varphi\right) \quad \text { in } R \backslash E
$$

Since $\varphi_{R}^{\wedge}-\varphi$ is continuous on $R$ and since the second term in the right-hand side is bounded in $R \backslash E$, it suffices to show that $\varphi_{R}^{\wedge}-\varphi_{R \backslash E}^{\wedge}$ is bounded in $R \backslash E$. To prove this we remark that there exists a sequence of domains $D=D_{E} \cup K_{E}$ in $R$ such that (a) $K_{E} \supset E$ is compact, (b) $\partial K_{E}$ consists of piecewise analytic Jordan curves, (c) $D_{E}=D \backslash K_{E} \uparrow R \backslash E$ as $D \uparrow R$. Then,

$$
\varphi_{D}^{\wedge}-\varphi_{D_{E}}^{\wedge}= \begin{cases}0 & \text { on } \partial D \\ \varphi_{D}^{\wedge}-\varphi & \text { on } \partial K_{E}\end{cases}
$$

so that

$$
\varphi_{D}^{\wedge}-\varphi_{D_{E}}^{\wedge} \leq \max _{K_{E}}\left(\varphi_{R}^{\wedge}-\varphi\right) \text { in } D_{E}
$$

Letting $D \uparrow R$ we now obtain

$$
\varphi_{R}^{\wedge}-\varphi_{R \backslash E}^{\wedge} \leq \max _{K}\left(\varphi_{R}^{\wedge}-\varphi\right) \quad \text { in } R \backslash E
$$

where $K$ is a compact set whose interior contains $E$.

REMARKS. We pose here for some references to $\mathrm{BMOA}_{e}(R)$ and $\operatorname{VMOA}_{e}(R)$.

(a) For $f \in M_{e}(R)$ pole-free, we have

$$
\Delta\left(|f(z)|^{2}\right) d x d y=4\left|f^{\prime}(z)\right|^{2} d x d y, \quad z \in R .
$$

By the Green formula it is now easy to prove the following.

(ai) $f$ is of Hardy class $H_{e}^{2}(R)$, that is, $|f|^{2}$ has a harmonic majorant on $R$ if and only if for a point $w \in R$,

$$
\iint_{R}\left|f^{\prime}(z)\right|^{2} g(z, w) d x d y<\infty
$$


(aii) $f \in \mathrm{BMOA}_{e}(R)$ if and only if $f \in H_{e}^{2}(R)$ and $\left(|f|^{2}\right)_{R}^{\wedge}-|f|^{2}$ is bounded there, while, $f \in \mathrm{VMOA}_{e}(R)$ if and only if $f \in H_{e}^{2}(R)$ and

$$
\lim _{w \rightarrow \partial R}\left[\left(|f|^{2}\right)_{R}^{\wedge}(w)-|f(w)|^{2}\right]=0 .
$$

The $\mathrm{BMOA}_{e}$ version of Corollary 3.3 is obvious.

(b) The analogue of Corollary 3.4 is valid.

If $E \subset R$ is compact and of capacity zero, and if $f \in \operatorname{BMOA}(R \backslash E)$, then there exists $F \in \operatorname{BMOA}(R)$ with $F \equiv f$ in $R \backslash E$.

The proof is essentially the same as that of Corollary 3.4. The existence of a single-valued $F \in H_{e}^{2}(R)$ with $F \equiv f$ in $R \backslash E$ follows from $\alpha=2$ in $[\mathbf{P}$, Théorème 20 , p. 182].

Returning to $f \in \mathrm{BC}_{e}(R), f \not \equiv 0$, we now consider $m_{S}(w, f)$ and $N(w, f)$; see $X(w, f)$ before Theorem 2.4. Then $m_{S}(w, f)$ is a nonnegative harmonic function of $w$ on $R$ and $N(w, f)=\sum g(w, b)$, the summation being extended over all the poles $b$ of $f$ in $R$, each counted with its order, is harmonic in $R$ minus the poles of $f$. Set

$$
\begin{aligned}
& F(w, f)=\exp \left[-m_{S}(w, f)-i m_{S}^{*}(w, f)\right], \\
& \beta(w, f)=\exp \left[-N(w, f)-i N^{*}(w, f)\right]
\end{aligned}
$$

for $w \in R$; both are bounded by one, and hence are in $\operatorname{UBC}_{e}(R)$ by the remark after Theorem 2.4. In particular, $\beta$ is a Blaschke product if $R=\Delta$.

The canonical decomposition, referred to in the title of the present section, is obtained by Sario [SN, Theorem 3B, p. 83 and Corollary 7, p. 86], which, for the clarity, we propose in the form of a lemma.

LEMMA 3.5. For $f \in \mathrm{BC}_{e}(R), f \not \equiv 0$, we have

$$
|f(w)|=\left|\frac{\beta(w, 1 / f) F(w, 1 / f)}{\beta(w, f) F(w, f)}\right| \quad \text { at each } w \in R .
$$

Note that $1 / f \in \mathrm{BC}_{e}(R)$. The following is an extension of $\left[\mathbf{Y}_{\mathbf{1}}\right.$, Corollary 4.1, p. 359].

THEOREM 3.6. If $f \in \mathrm{UBC}_{e}(R), f \neq \equiv 0$, then $F(w, 1 / f) / F(w, f) \in \mathrm{UBC}_{e}(R)$.

It is known that the converse is false for $R=\Delta$; see $\left[\mathbf{Y}_{\mathbf{1}}\right.$, p. 359]. Further it is known that $\operatorname{UBC}(\Delta)$ is not closed for multiplication and summation $\left[\mathbf{Y}_{\mathbf{1}}\right.$, Theorem 4.2 , p. 359].

ProOF OF THEOREM 3.6. To prove first that

$$
\beta(w, f) f(w) \in \mathrm{UBC}_{e}(R),
$$

we set

$$
\beta(w)=\beta(w, f), \quad h(w)=\beta(w) f(w), \quad w \in R .
$$

Then $\beta \in \mathrm{BC}_{e}$ and $h \in \mathrm{BC}_{e}$ is pole-free. First we consider those $w \in R$ for which $|f(w)| \neq \infty$. It follows from (2.7) of Theorem 2.4 that

$$
\begin{aligned}
m(w, f) & =T(w, f)+\log |\beta(w)|+\frac{1}{2} \log \left(1+|f(w)|^{2}\right) \\
& =T(w, f)+\frac{1}{2} \log \left(|\beta(w)|^{2}+|h(w)|^{2}\right) .
\end{aligned}
$$


Since

$$
\begin{aligned}
& m(w, h) \leq m(w, \beta)+m(w, f), \quad \text { and } \\
& A \equiv\left(|\beta(w)|^{2}+|h(w)|^{2}\right) /\left(1+|h(w)|^{2}\right) \leq 1,
\end{aligned}
$$

it again follows from $(2.7)$ that

$$
\begin{aligned}
T(w, h) & =m(w, h)-\frac{1}{2} \log \left(1+|h(w)|^{2}\right) \\
& \leq m(w, \beta)+m(w, f)-\frac{1}{2} \log \left(1+|h(w)|^{2}\right) \\
& \leq \frac{1}{2} \log 2+T(w, f)+\frac{1}{2} \log A \\
& \leq \frac{1}{2} \log 2+T(w, f) .
\end{aligned}
$$

Therefore,

$$
T(w, h) \leq \frac{1}{2} \log 2+\sup _{\zeta \in R} T(\zeta, f)
$$

for $w \in R$ with $|f(w)| \neq \infty$. Since $T(w, h)$ is continuous on $R$, and since the poles of $f$ are isolated, we observe that $\sup T(w, h)$ for $w \in R$ does not exceed the right-hand side of (3.9), which completes the proof of (3.8).

Now, $1 / h \in \mathrm{UBC}_{e}(R)$ by (3.8). Apply (3.8) to $1 / h$ instead of $f$. Since $|\beta(w, 1 / f)|$ $=|\beta(w, 1 / h)|$, we conclude that $F(w, f) / F(w, 1 / f) \in \mathrm{UBC}_{e}(R)$ because

$$
|F(w, f) / F(w, 1 / f)|=|\beta(w, 1 / h)(1 / h(w))| .
$$

Thus, $F(w, 1 / f) / F(w, f) \in \mathrm{UBC}_{e}(R)$.

4. Counting function. For nonconstant $f \in M(R), w \in D$, and $z \in \mathbf{C}^{*}$ we define

$$
N_{S}(D, w, z, f)=N_{S}(D, w, 1 /(f-z)) \quad \text { and } \quad N(D, w, z, f)=N(D, w, 1 /(f-z)) \text {. }
$$

Then $N(D, w, z, f)=\sum g_{D}(w, \zeta)$, where the sum is extended over all $z$-points $\zeta$ of $f$ in $D$, each counted with its order. Further, set

$$
\begin{aligned}
& N_{S}(w, z, f)=\lim _{D \uparrow R} N_{S}(D, w, z, f) \quad \text { and } \\
& N(w, z, f)=\lim _{D \uparrow R} N(D, w, z, f)=\sum g(w, \varsigma),
\end{aligned}
$$

where the sum is extended over all $z$-points $\zeta$ of $f$ in $R$, each counted with its order. Note that $N(w, z, f)=G(w, z, f)$ in [SN, p. 90]. Apparently, $N(w, z, f)=\infty$ if $f(w)=z$. Heins $\left[\mathbf{H}_{2}\right]$ called $f \in M(R)$ a Lindelöfian map from $R$ into $\mathbf{C}^{*}$, or $f$ is Lindelöfian and meromorphic, if $N(w, z, f)<\infty$ for each pair $z \in \mathbf{C}^{*}, w \in R$, with $f(w) \neq z$. It is known that $f \in \mathrm{BC}(R)$ if and only if $f$ is Lindelöfian and meromorphic [SN, Theorem 6E, p. 92].

As usual we consider the chordal distance

$$
\chi(a, b)=|a-b| /\left[\left(1+|a|^{2}\right)\left(1+|b|^{2}\right)\right]^{1 / 2}, \quad a, b \in \mathbf{C}^{*},
$$

with the obvious change for $a=\infty$ or $b=\infty$. The length of a curve on $\mathbf{C}^{*}$ measured by $d \chi(\varsigma)=|d \varsigma| /\left(1+|\zeta|^{2}\right), \varsigma \in \mathbf{C}^{*}$, is the same as its Euclidean length, considered as a curve on the Riemann sphere in the Euclidean space. Set

$$
\Gamma(a, \rho)=\left\{z \in \mathbf{C}^{*} ; \chi(z, a)=\rho\right\}, \quad a \in \mathbf{C}^{*}, 0<\rho<1,
$$

and set, for $w \in R, 0<\rho<1$, and $f \in M(R)$,

$$
C(w, \rho, f)=\sup _{z \in \Gamma(f(w), \rho)} N(w, z, f) .
$$

We begin with criteria for $f \in M(R)$ to be of $\mathrm{BC}(R)$ in terms of $C$. 
THEOREM 4.1. For $f \in M(R)$ the following are mutually equivalent.

(I) $f \in \mathrm{BC}(R)$.

(II) There exists a pair $w, \rho$, with $w \in R, 0<\rho<1$, such that $C(w, \rho, f)<\infty$.

(III) For each pair $w, \rho$ with $w \in R, 0<\rho<1$, we have $C(w, \rho, f)<\infty$.

A weaker condition than (II) implies (I). Actually, if $N(w, z, f)<\infty$ for a set of $z \in \mathbf{C}^{*}$ of positive capacity (see $\S 5$ for the definition of "capacity" of a set on $\left.\mathbf{C}^{*}\right)$, then $f \in \mathrm{BC}(R)$ by $[\mathbf{P}$, Théorème 22, p. 190]; the set $\Gamma(f(w), \rho)$ is of positive capacity. A reason of proposing (II) is to compare it with (V) in

THEOREM 4.2. For $f \in M(R)$ the following are mutually equivalent.

(IV) $f \in \mathrm{UBC}(R)$.

(V) There exists $\rho, 0<\rho<1$, such that $\sup _{w \in R} C(w, \rho, f)<\infty$.

(VI) For each $\rho, 0<\rho<1$, $\sup _{w \in R} C(w, \rho, f)<\infty$.

Postponing the proofs of Theorems 4.1 and 4.2 we first note that the length of $\Gamma(a, \rho)$ is independent of $a \in \mathbf{C}^{*}$ and is

$$
l(\rho)=2 \pi \rho\left(1-\rho^{2}\right)^{1 / 2}, \quad 0<\rho<1 ;
$$

for example, the length of the equator $\Gamma(0,1 / \sqrt{2})=\Gamma(\infty, 1 / \sqrt{2})$ is $l(1 / \sqrt{2})=\pi$. Set

$$
c(\rho)=\max \left[\rho^{-1}\left(1-\rho^{2}\right)^{1 / 2}, \rho\left(1-\rho^{2}\right)^{-1 / 2}\right], \quad 0<\rho<1 .
$$

THEOREM 4.3. The following estimates hold for $f \in M(R), 0<\rho<1$, and $w \in R$.

(VII) $c(\rho)^{2} T(w, f) \geq l(\rho)^{-1} \int_{\Gamma(f(w), \rho)} N(w, z, f) d \chi(z)-(1 / 2) \log 2$.

(VIII) $c(\rho)^{-2} T(w, f) \leq l(\rho)^{-1} \int_{\Gamma(f(w), \rho)} N(w, z, f) d \chi(z)+(1 / 2) \log 2$.

The estimates (VII) and (VIII) are motivated by the celebrated Cartan formula [SN, (56), p. 89]:

$$
T_{S}(D, w, f)=\log ^{+}|f(w)|+(2 \pi)^{-1} \int_{0}^{2 \pi} N_{S}\left(D, w, e^{i t}, f\right) d t
$$

for $f \in M(R)$, provided that $f(w) \neq \infty$. A merit of (VII) and (VIII) might be that the right-hand sides have no term like $\log ^{+}|f(w)|$; also no assumption on the value $f(w)$ is posed.

With the aid of (VIII) of Theorem 4.3, (II) $\Rightarrow$ (I) of Theorem 4.1 and (V) $\Rightarrow$ (IV) of Theorem 4.2 are immediately obtained.

PROOF OF THEOREM 4.3. We may suppose that $f$ is nonconstant. We shall use the following abbreviation like (2.10):

$$
N_{f}(z)=N(D, w, z, f) \text {. }
$$

Set

$$
\delta=\rho\left(1-\rho^{2}\right)^{-1 / 2},
$$

so that $l(\rho)=2 \pi \delta\left(1+\delta^{2}\right)^{-1}$ by (4.1) and $c(\rho)=\max \left(\delta, \delta^{-1}\right)$ by (4.2). We consider $h \in M(R)$ defined by

$$
h=(f-f(w)) /(1+\overline{f(w)} f) \quad(=1 / f \text { if } f(w)=\infty) .
$$


First we observe that

$$
(2 \pi)^{-1} \int_{0}^{2 \pi} N_{h}\left(\delta e^{i t}\right) d t=l(\rho)^{-1} \int_{\Gamma(f(w), \rho)} N_{f}(z) d \chi(z) .
$$

For the proof we note that the Möbius transformation

$$
\varsigma=(z-f(w)) /(1+\overline{f(w)} z)
$$

maps the circle $\Gamma(f(w), \rho)$ one-to-one onto the circle $\{|\zeta|=\delta\}$ and further,

$$
|d \zeta|=\left(1+\delta^{2}\right) d \chi(z) \quad \text { for } z \in \Gamma(f(w), \rho) .
$$

Now, the left-hand side of (4.6), denoted by $A$, is expressed as

$$
A=(2 \pi \delta)^{-1} \int_{|\zeta|=\delta} N_{h}(\zeta)|d \zeta|
$$

which yields (4.6).

Next we claim that

$$
|T(h / \delta)-A| \leq \frac{1}{2} \log 2
$$

Since $h(w)=0$, it follows from (2.16) for $h / \delta$ instead of $f$ that

$$
\left|T(h / \delta)-T_{S}(h / \delta)\right| \leq \frac{1}{2} \log 2 .
$$

We now remember the identity

$$
(2 \pi)^{-1} \int_{0}^{2 \pi} \log \left|b-\delta e^{i t}\right| d t=\log \delta+\log ^{+}|b / \delta|
$$

for each $b \in \mathbf{C}$; see $[\mathbf{N}$, p. 178] for the calculation. Jensen's formula [SN, (2), p. 76], applied to $h-\delta e^{i t}$ with $h(w)-\delta e^{i t}=\delta e^{i t}$, yields

$$
\log \delta=-(2 \pi)^{-1} \int_{\partial D} \log \left|h(z)-\delta e^{i t}\right| d g_{D}^{*}(z, w)+N(h)-N_{h}\left(\delta e^{i t}\right)
$$

for each $t \in[0,2 \pi)$. Calculating the integral means of both sides of (4.10) with respect to $d t$ in $[0,2 \pi)$, and observing (4.9), together with $N(h)=N(h / \delta)$, we now obtain

$$
\log \delta=\log \delta+m_{S}(h / \delta)+N(h / \delta)-A,
$$

whence $T_{S}(h / \delta)=A$. The estimate $(4,7)$ follows then from (4.8).

Finally for $c=c(\rho)$, some computations yield $c^{-1} h^{\#} \leq(h / \delta)^{\#} \leq c h^{\#}$. Since $T(h)=T(f)$, it follows that $c^{-2} T(f) \leq T(h / \delta) \leq c^{2} T(f)$.

The estimates (VII) and (VIII) for $R=D$ now follow from the above, together with (4.6) and (4.7). Letting $D \uparrow R$ we arrive at the requested conclusions.

For the remaining proofs of Theorems 4.1 and 4.2 we prove

LEMMA 4.4. Let $f \in M(R), 0<\rho<1, w \in R$, and $1<q<\infty$. Then (IX) $C(w, \rho, f) \leq q c(\rho)^{2} T(w, f)+(q / 2) \log 2+\log [(q+1) /(q-1)]$.

On setting $q=2$, say, we see that (I) $\Rightarrow$ (III) of Theorem 4.1 and (IV) $\Rightarrow$ (VI) of Theorem 4.2 follow from (IX). Since (III) $\Rightarrow($ II $)$ and (VI) $\Rightarrow(\mathrm{V})$ are trivial, this completes the proofs of Theorems 4.1 and 4.2 . 
Proof OF Lemma 4.4. Fix $D, w \in D$. As is noted by O. Lehto $[\mathbf{L}]$ the function $N_{f}(z)$ (see (4.3)) of $z \in \mathbf{C}^{*}$ is subharmonic in $\mathbf{C}^{*} \backslash\{f(w)\}$ and $N_{f}$ has the logarithmic singularity at $f(w)$ in the sense that

$$
N_{f}(z)+\log |z-f(w)| \quad\left(N_{f}(z)-\log |z| \text { if } f(w)=\infty\right)
$$

is subharmonic in $\mathbf{C}^{*} \backslash\{-1 / \overline{f(w)}\}$. Thus,

$$
A^{\prime} \equiv \sup _{\chi(z, f(w)) \geq \rho} N_{f}(z)=\sup _{z \in \Gamma(f(w), \rho)} N_{f}(z) .
$$

Our task is therefore to show that

$$
A^{\prime} \leq q c(\rho)^{2} T(f)+(q / 2) \log 2+\log [(q+1) /(q-1)] .
$$

Since $T(w, f) \geq T(f)$ and since $N_{f} \uparrow N(w, f)$ as $D \uparrow R$, (IX) follows from (4.12).

We consider again $h$ of (4.5) for which $h(w)=0$. Then $u(z) \equiv N_{h}(1 / z)=$ $N_{1 / h}(z)$ is subharmonic in $\mathbf{C}$ and $u(z)-\log |z|$ is subharmonic in $\mathbf{C}^{*} \backslash\{0\}$. By (4.6) and (VII) of Theorem 4.3 we obtain

$$
\begin{aligned}
(2 \pi)^{-1} & \int_{0}^{2 \pi} u\left(\delta^{-1} e^{i t}\right) d t=(2 \pi)^{-1} \int_{0}^{2 \pi} u\left(\delta^{-1} e^{-i t}\right) d t \\
= & (2 \pi)^{-1} \int_{0}^{2 \pi} N_{h}\left(\delta e^{i t}\right) d t \leq c(\rho)^{2} T(f)+\frac{1}{2} \log 2 \equiv \alpha,
\end{aligned}
$$

where $\delta$ is defined in (4.4).

Our aim is to prove that

$$
|z| \leq \delta^{-1} \Rightarrow u(z) \leq q \alpha+\log \lambda, \quad \lambda=(q+1) /(q-1) .
$$

Then, the identities

$$
A^{\prime}=\sup _{\delta \leq|z| \leq \infty} N_{h}(z)=\sup _{|z| \leq \delta^{-1}} u(z),
$$

together with (4.14), show (4.12).

Let $u_{\delta}^{\wedge}$ be the least harmonic majorant of $u$ in the disk $|z|<\delta^{-1}$, which can be expressed by the Poisson formula,

$$
u_{\delta}^{\wedge}(z)=(2 \pi)^{-1} \int_{0}^{2 \pi} \frac{\delta^{-2}-|z|^{2}}{\left|\delta^{-1} e^{i t}-z\right|^{2}} u\left(\delta^{-1} e^{i t}\right) d t, \quad|z|<\delta^{-1} .
$$

Set $\Lambda=\lambda^{-1} \delta^{-1}$. Then, on the circle $|z|=\Lambda$ we have the estimate

$$
u_{\delta}^{\wedge}(z) \leq \frac{\Lambda^{-1}+\delta}{\Lambda^{-1}-\delta}(2 \pi)^{-1} \int_{0}^{2 \pi} u\left(\delta^{-1} e^{i t}\right) d t
$$

whence, by (4.13), we obtain

$$
\max _{|z|=\Lambda} u_{\delta}^{\wedge}(z) \leq q \alpha .
$$

The maximum principle now yields

$$
|z| \leq \Lambda \Rightarrow u(z) \leq u_{\delta}^{\wedge}(z) \leq q \alpha .
$$

On the other hand, by the maximum principle for $u(z)-\log |z|$, with (4.15), we have

$$
\begin{aligned}
\Lambda<|z|<\infty \Rightarrow u(z)-\log |z| & \leq \sup _{|\varsigma|=\Lambda}(u(\varsigma)-\log |\varsigma|) \\
& \leq q \alpha-\log \Lambda .
\end{aligned}
$$


Therefore,

$$
\begin{aligned}
\Lambda<|z| \leq \delta^{-1} \Rightarrow u(z) & \leq q \alpha-\log \Lambda+\log \delta^{-1} \\
& =q \alpha+\log \lambda .
\end{aligned}
$$

The estimate (4.14) now follows from (4.16) and (4.17).

5. Uniformization. For a projection map $\pi: \Delta \rightarrow R$, there exists the group $\mathcal{G}$ of conformal homeomorphisms from $\Delta$ onto $\Delta$, called the covering transformation group, such that $\pi \circ \gamma=\pi$ for each $\gamma \in \mathcal{G}$. Thus, for $f \in M(R)$, the function $f \circ \pi$ is $\mathcal{G}$-automorphic in the sense that $(f \circ \pi) \circ \gamma=f \circ \pi$ for all $\gamma \in \mathcal{G}$. We begin with

THEOREM 5.1. For each $f \in M(R)$ and each $\delta \in \Delta$, we have

$$
T(R, \pi(\delta), f)=T(\Delta, \delta, f \circ \pi) .
$$

Set

$$
\|f\|_{\mathrm{UBC}(R)}=\sup _{w \in R} T(R, w, f)
$$

for $f \in M(R)$. Besides the obvious consequence of Theorem 5.1 that $f \in \mathrm{BC}(R)$ if and only if $f \circ \pi \in \mathrm{BC}(\Delta)$, we have

COROLlaRY 5.2. For $f \in M(R)$, the equality

$$
\|f\|_{\mathrm{UBC}(R)}=\|f \circ \pi\|_{\mathrm{UBC}(\Delta)}
$$

holds. Thus, $f \in \mathrm{UBC}(R)$ if and only if $f \circ \pi \in \mathrm{UBC}(\Delta)$.

A subset $A$ of $\mathbf{C}^{*}$ is said to be of positive capacity if $A$ contains a closed subset of $\mathbf{C}^{*}$ of positive elliptic capacity [T, p. 90], or equivalently, $A \backslash\{\infty\}$ contains a bounded (in $\mathbf{C}$ ) and closed set of positive capacity [T, p. 55].

COROLlaRY 5.3. Suppose that for $f \in M(R)$, the exceptional set $\mathbf{C}^{*} \backslash f(R)$ is of positive capacity. Then $f \in \mathrm{UBC}(R)$.

Applying the known theorem $\left[\mathbf{Y}_{\mathbf{2}}\right.$, Theorem 1] to $f \circ \pi$ we observe that $f \circ \pi \in$ $\mathrm{UBC}(\Delta)$, whence $f \in \operatorname{UBC}(R)$.

COROllary 5.4. Suppose that a subdomain $G$ of $\mathrm{C}^{*}$ has the projection $\pi: \Delta \rightarrow$ $G$. Then, $\pi \in \mathrm{UBC}(\Delta)$ if and only if $\mathbf{C}^{*} \backslash G$ is of positive capacity.

The "only if" part is a consequence of $[\mathbf{N}$, Satz 1, p. 213] because $\pi \in \mathrm{BC}(\Delta)$. The "if" part is a consequence of Corollary 5.3.

PROOF OF THEOREM 5.1. We note that, by the construction of $f_{1}$ and $f_{2}$ in Lemma 3.0, both are members of $M$. Thus,

$$
f \circ \pi=F_{1} / F_{2}, \quad F_{k}=f_{k} \circ \pi, \quad k=1,2,
$$

yields a canonical decomposition of $f \circ \pi$ in $\Delta$. For $\varphi$ of (3.3) we set

$$
\Phi=\varphi \circ \pi=\frac{1}{2} \log \left(\left|F_{1}\right|^{2}+\left|F_{2}\right|^{2}\right) .
$$

We shall show that

$$
\Phi_{\Delta}^{\wedge}-\Phi=\left(\varphi_{R}^{\wedge}-\varphi\right) \circ \pi
$$

that $\Phi_{\Delta}^{\wedge}$ exists if and only if $\varphi_{R}^{\wedge}$ exists is clear in the following context. The identity (5.1) now follows from (3.5) with (5.4). 
For the proof of (5.4) it suffices to observe that $\Phi_{\Delta}^{\wedge}=\Phi_{R}^{\wedge} \circ \pi$. Since $\varphi \leq \varphi_{R}^{\wedge}$, it follows that $\Phi \leq \varphi_{R}^{\wedge} \circ \pi$, whence $\Phi_{\Delta}^{\wedge} \leq \varphi_{R}^{\wedge} \circ \pi$. To prove the inverse we remark that $\Phi_{\Delta}^{\wedge}$ is $\mathcal{G}$-automorphic by (5.3). Then the function $\psi$ on $R$ well-defined by

$$
\psi(z)=\Phi_{\Delta}^{\wedge}(\varsigma) \text { for } z \in R \text { with } \pi(\zeta)=z, \zeta \in \Delta,
$$

is harmonic on $R$. Therefore, it follows from $\Phi_{\Delta}^{\wedge} \geq \Phi=\varphi \circ \pi$ that $\psi \geq \varphi$, whence $\psi \geq \varphi_{R}^{\wedge}$, so that $\Phi_{\Delta}^{\wedge} \geq \varphi_{R}^{\wedge} \circ \pi$ in $\Delta$.

We now extend [ $\mathbf{Y}_{\mathbf{2}}$, Theorem 3]. Let $S$ be a hyperbolic Riemann surface with the Green functions $g_{S}(z, w)$. An analytic mapping $h: R \rightarrow S$ is said to be of type $B l$ (see $\left[\mathbf{H}_{\mathbf{1}}\right]$ ) if for each $w \in S$, the function $g_{S}(h(z), w)-\sum g_{R}(z, \zeta)$ of $z \in R$ is singular, that is, it does not dominate any strictly positive and bounded harmonic function on $R$, where the summation is taken over all roots $\zeta \in R$ of the equation $h=w$, counted with their multiplicities.

THEOREM 5.5. The following hold for $f \in M(S)$.

(I) If $f \in \operatorname{UBC}(S)$, then for each analytic map $h: R \rightarrow S$, we have

$$
\|f \circ h\|_{\mathrm{UBC}(R)} \leq\|f\|_{\mathrm{UBC}(S)},
$$

so that $f \circ h \in \mathrm{UBC}(R)$.

(II) If $h: R \rightarrow S$ is of type $B l$, and if $f \circ h \in \mathrm{UBC}(R)$, then

$$
\|f \circ h\|_{\mathrm{UBC}(R)}=\|f\|_{\mathrm{UBC}(S)},
$$

so that $f \in \mathrm{UBC}(S)$.

PROOF. Let $\pi_{R}: \Delta \rightarrow R$ and $\pi_{S}: \Delta \rightarrow S$ be the projection maps. Then a branch of $\pi_{S}^{-1} \circ h \circ \pi_{R}$ is single-valued in $\Delta$, which we denote simply, $H=\pi_{S}^{-1} \circ h \circ \pi_{R}$. Set $F=f \circ \pi_{S}$, so that $f \circ h \circ \pi_{R}=F \circ H$. If $f \in \operatorname{UBC}(S)$, then $F \in \operatorname{UBC}(\Delta)$ with

$$
\|F\|_{\mathrm{UBC}(\Delta)}=\|f\|_{\mathrm{UBC}(S)}
$$

by Corollary 5.2. On the other hand, it follows from $\left[\mathbf{Y}_{\mathbf{2}}\right.$, Theorem 3, (I)] that

$$
\|F \circ H\|_{\mathrm{UBC}(\Delta)} \leq\|F\|_{\mathrm{UBC}(\Delta)} \text {. }
$$

Corollary 5.2 again shows that

$$
\|F \circ H\|_{\mathrm{UBC}(\Delta)}=\|f \circ h\|_{\mathrm{UBC}(R)},
$$

so that (5.5) follows from (5.7), (5.8), and (5.9).

Now, if $h$ is of type $B l$, then $H$ is of type $B l$ by [ $\mathbf{H}_{\mathbf{1}}$, Corollary, p. 472] because $S$ is hyperbolic. Thus, if $f \circ h \in \mathrm{UBC}(R)$, then by (5.9), $F \circ H \in \mathrm{UBC}(\Delta)$, so that, by $\left[\mathbf{Y}_{\mathbf{2}}\right.$, Theorem 3, (II)] we have $\|F \circ H\|_{\mathrm{UBC}(\Delta)}=\|F\|_{\mathrm{UBC}(\Delta)}$, which, together with (5.7) and (5.9), yields (5.6).

REMARKS. (a) Is it true that $f \in \mathrm{UBC}_{0}(R)$ if and only if $f \circ \pi \in \mathrm{UBC}_{0}(\Delta)$ ? This is open, as far as the author knows. The similar question for VMOA is also open.

(b) Let $N(R)$ denote the family of normal meromorphic functions on $R$; namely, $f \in N(R)$ if and only if there is a constant $c>0$ such that $f^{\#}(z) \leq c \sigma_{R}(z), z \in R$, where $\sigma_{R}(z)|d z|$ is the hyperbolic metric on $R$; note that $\sigma_{\Delta}(z)=\left(1-|z|^{2}\right)^{-1}$. It is easy to observe that $f \in N(R)$ if and only if $f \circ \pi \in N(\Delta)$. It then follows from the known inclusion formula $\operatorname{UBC}(\Delta) \subset N(\Delta)\left[\mathbf{Y}_{\mathbf{1}}\right.$, Theorem 3.1] that $\operatorname{UBC}(R) \subset$ $N(R)$. 
(c) By the remarks after the proof of Corollary 3.4, we can prove the analogue of $(5.1)$ for pole-free $f \in M(R)$ by the similar method. Namely,

$$
\iint_{R}\left|f^{\prime}(z)\right|^{2} g(z, \pi(\delta)) d x d y=\iint_{\Delta}\left|(f \circ \pi)^{\prime}(z)\right|^{2} g_{\Delta}(z, \delta) d x d y
$$

Metzger $[\mathbf{M}]$ obtained this by analyzing the Green functions.

(d) Define the BMOA norm for pole-free $f \in M(R)$ by

$$
\|f\|_{\mathrm{BMOA}(R)}=\sup _{w \in R} \iint_{R}\left|f^{\prime}(z)\right|^{2} g(z, w) d x d y .
$$

The BMOA analogue of (5.2) is then $\|f\|_{\mathrm{BMOA}(R)}=\|f \circ \pi\|_{\mathrm{BMOA}(\Delta)}$.

(e) A BMOA analogue of Theorem 5.5 is valid. Instead of [ $\mathbf{Y}_{\mathbf{2}}$, Theorem 3] we use the corresponding one found by K. Stephenson [St, Theorem 3, p. 572, in particular]. The results are:

If $f \in \operatorname{BMOA}(S)$, then

$$
\|f \circ h\|_{\mathrm{BMOA}(R)} \leq\|f\|_{\mathrm{BMOA}(S)}
$$

for each analytic map $h: R \rightarrow S$.

If $h: R \rightarrow S$ is of type $B l$, and if $f \circ h \in \mathrm{BMOA}(R)$, then $\|f \circ h\|_{\mathrm{BMOA}(R)}=$ $\|f\|_{\mathrm{BMOA}(S)}$, so that $f \in \operatorname{BMOA}(S)$.

(f) Metzger $[\mathbf{M}]$ introduced the family $O_{\mathrm{BMOA}}$ of Riemann surfaces on which BMOA consists only of constants. By an obvious reason we include Riemann surfaces of $O_{G}$ in $O_{\mathrm{BMOA}}$. Let $O_{\mathrm{UBC}}$ be the family of Riemann surfaces which are either of $O_{G}$ or admit no nonconstant UBC functions. We shall prove the strict inclusion formula

$$
O_{\mathrm{UBC}} \varsubsetneqq O_{\mathrm{BMOA}} .
$$

Our work should be finding $R \in O_{\mathrm{BMOA}} \backslash O_{\mathrm{UBC}}$. Let $E$ be a compact set of linear measure zero, yet of positive capacity lying on the real axis. Then $R=\mathbf{C}^{*} \backslash E$ is the desired. Since the function $z$ is of $\operatorname{UBC}(R)$ by Corollary 5.3 , it follows that $R \notin O_{\mathrm{UBC}}$. On the other hand, each $f \in \operatorname{BMOA}(R)$ is of class $H^{2}(R)$, that is, $|f|^{2}$ has a harmonic majorant on $R$. It is familiar (see, for example, [ $\mathbf{Y}_{\mathbf{3}}$, p. 334]) that $f$ can be extended holomorphically to $\mathbf{C}^{*}$, so that, $f$ must be a constant. Thus $R \in O_{\mathrm{BMOA}}$.

(g) Let $\operatorname{UBCA}(\Delta)$ be the set of all pole-free members of $\operatorname{UBC}(\Delta)$. It is apparent that $\operatorname{BMOA}(\Delta) \subset \operatorname{UBCA}(\Delta)$. On observing $[\mathbf{B}$, Corollary 2, p. 15], one might suspect that $\operatorname{BMOA}(\Delta)=\operatorname{UBCA}(\Delta)$. This is not the case. Let

$$
f(z)=(1+z) /(1-z) .
$$

By Corollary $5.3, f \in \operatorname{UBCA}(\Delta)$, yet $f \notin \operatorname{BMOA}(\Delta)$ because $f$ is not Bloch in the sense that $\left(1-|z|^{2}\right)\left|f^{\prime}(z)\right|$ is unbounded in $\Delta$.

\section{REFERENCES}

[B] Albert Baernstein II, Analytic functions of bounded mean oscillation, Aspects of Contemporary Complex Analysis (D. A. Brannan and J. G. Clunie, eds.), Academic Press, London, 1980, pp. 3-36.

[F] Herta Florack, Reguläre und meromorphe Funktionen auf nicht geschlossenen Riemannschen Flächen, Schriftenreihe des Mathematischen Instituts der Universität Münster, Heft 1, Max Kramer, Münster, 1948. 
[H1] Maurice Heins, On the Lindelö principle, Ann. of Math. 61 (1955), 440-473.

$\left[\mathbf{H}_{2}\right]$ _ Lindelöfian maps, Ann. of Math. 62 (1955), 418-446.

[L] Olli Lehto, A majorant principle in the theory of functions, Math. Scand. 1 (1953), 5-17.

[M] Thomas Metzger, On BMOA for Riemann surfaces, Cainad. J. Math. 33 (1981), 1255-1260.

[N] Rolf Nevanlinna, Eindeutige analytische Funktionen, 2nd ed., Springer-Verlag, Berlin, Göttingen and Heidelberg, 1953.

[P] Michel Parreau, Sur les moyennes des fonctions harmoniques et analytiques et la classification des surfaces de Riemann, Ann. Inst. Fourier (Grenoble) 3 (1951), 103-197.

[S1] Leo Sario, Second main theorem without exceptional intervals on arbitrary Riemann surfaces, Michigan Math. J. 10 (1963), 207-219.

[S $\left.\mathbf{S}_{\mathbf{2}}\right]$, On locally meromorphic functions with single-valued moduli, Pacific J. Math. 13 (1963), 709-724.

[St] Kenneth Stephenson, Weak subordination and stable classes of meromorphic functions, Trans. Amer. Math. Soc. 262 (1980), 565-577.

[SN] Leo Sario and Kiyoshi Noshiro, Value distribution theory, Van Nostrand, Princeton, N.J., 1966.

[T] Masatsugu Tsuji, Potential theory in modern function theory, Maruzen, Tokyo, 1959.

[Y 1 ] Shinji Yamashita, Functions of uniformly bounded characteristic, Ann. Acad. Sci. Fenn. Ser. A I Math. 7 (1982), 349-367.

$\left[\mathbf{Y}_{\mathbf{2}}\right] \ldots$, Exceptional sets, subordination, and functions of uniformly bounded characteristic, Math. Scand. (to appear).

[Y $\left.\mathbf{Y}_{3}\right]$ _ Some remarks on analytic continuations, Tôhoku Math. J. 21 (1969), 328-335.

Department of Mathematics, Tokyo Metropolitan University, Fukasawa, SETAGAYA, TOKYO 158 , JAPAN 